Available online at GSC Online Press Directory

GSC Biological and Pharmaceutical Sciences

e-ISSN: 2581-3250, CODEN (USA): GBPSC2

Journal homepage: https://www.gsconlinepress.com/journals/gscbps

(RESEARCH ARTICLE)

\title{
Biological study on the impact of commonly used commercial fats and oil and threats of atherosclerosis
}

\author{
Javaid Farwa ${ }^{1}$, Zahoor Tahir ${ }^{1}$, Nazeer Sidra ${ }^{1}$, Ramzan Uzma ${ }^{2, *}$ and Matloob Asmat ${ }^{1}$ \\ ${ }^{1}$ National Institute of Food Science and Technology, University of Agriculture, Faisalabad, Pakistan. \\ ${ }^{2}$ Department of Zoology, University of the Punjab Lahore, Pakistan.
}

Publication history: Received on 07 July 2019; revised on 24 July 2019; accepted on 27 July 2019

Article DOI: https://doi.org/10.30574/gscbps.2019.8.1.0123

\begin{abstract}
Poor health due to suboptimal nutrition influences on many diseases including diabetes mellitus, obesity, cardiovascular diseases (CVD), elevated cholesterol and blood pressure (BP). World health organization estimated that mortality rate and disease burden due to CVD was around 17.3 million. The prevalence rate of coronary artery diseases (CAD) is higher in Pakistan that is 30\% of people age above 45 years. The objective of study involves evaluation of commonly used fats and oil to investigate its effect on lipid profile through in vivo studies. Hypercholesterolaemia was induced by feeding high cholesterol diet. Thirty Sprague dawdle rats were taken having five rats in each group. Margarine, butter, vanaspati ghee and olive oil were given with a specific dose $10 \mathrm{~g} / 100 \mathrm{~g}$ feed given 6 weeks on hypercholesterolaemic rats. Maximum weight gain was observed in G2, G3, G4 fed; margarine, butter, vanaspati ghee respectively. And maximum reduction in weight was observed in G5 fed on olive oil. Feeding cholesterol diet showed elevated level of lipid profile in G1. Coincident giving various fats and oil along with high cholesterol diet caused a highly significant increase in serum total cholesterol, low density lipoprotein (LDL), triglycerides except G5. Maximum increase of cholesterol was observed in groups fed butter, margarine, vanaspati ghee were recorded as $177.6 \mathrm{mg} / \mathrm{dL}, 185.5 \mathrm{mg} / \mathrm{dL}, 180.7 \mathrm{mg} / \mathrm{dL}$. Minimum reduction in cholesterol was recorded in olive oil group as $170.2 \mathrm{mg} / \mathrm{dL}$. Results suggested that the addition of fats showed a negative influence on lipid profile in hypercholesterolaemic rats.
\end{abstract}

Keywords: Health disorders; Atherosclerosis; Hypercholesterolemia; Fats and oil; Diseases; Prevention

\section{Introduction}

Atherosclerosis is the most prominent root of cardiovascular diseases (CVD). Risk of cardiovascular diseases has multifactor symptoms with combination from both inflammatory and lipid metabolism that affects vascular functions. Hypercholesterolaemia is a disease in which level of flowing low density lipoprotein (LDL) in blood is increased. Usually it is known as main modifiable risk factor of CVD. Although, little particles consider that it is more atherogenic because it is more susceptible to oxidation then it increases the rate of macrophages [1]. Cardiovascular diseases (CVD) are main public health issue and major cause of mortality and morbidity in western world. The major cause of metabolic syndrome, such as hypertension, obesity and diabetes is due to elevated consumption of fats. Several evidence have recognized that cholesterol rich diet including intermediate density lipoprotein (IDL), very low density lipoproteins (VLDL), apolioprotein B (Apo-B), and their constituent directly related in the formation of atherosclerotic cardiovascular diseases (ASCVD) [2]. In recent years dietary fatty acids have become a central theme in nutrition research. Fat in diet is essential but in limited amount for human body to survive. Energy provided by $1 \mathrm{~g}$ fat is $9 \mathrm{Kcal}$ while $4 \mathrm{Kcal}$ provided from carbohydrate and protein.

\footnotetext{
${ }^{*}$ Corresponding author

E-mail address: uzmaramzan99@gmail.com
} 
Fat that taken from diet is required for the absorption of fat soluble vitamins, hormones and major building block of cell membrane. Fat is very important for our survival but its consumption should limited otherwise it may linked to many diseases onset like obesity and coronary heart disease (CHD). The edible oils of interest in our study were olive oil contains monounsaturated fatty acids also have higher quantity of antioxidants. This oil involves in numerous biological functions which have beneficial effect on our health. Olive oil scavenges free radicals and has enough for inflammation and auto immune diseases prevention [3]. Olive oil have greater amount of MUFA than any other oil or fat. Many researches done on different oils and fats used in diet such as butter, olive oil and coconut oil and they increases CVD risk factors in men and women. Butter contain (66\% saturated fat) which is about 40\% palmitic (16:0) and stearic (18:0) acids, that high in saturated fat and it increases the cholesterol in blood. Consumption of higher quantity of excessive saturated fatty acids (SFA) increases lipid storage and inflammation that is related with the threat of inflammatory cardiovascular diseases. In a current study, based on hydrogenated oils, trans fatty acid (TFA) may elevate blood cholesterol as compared to non-hydrogenated oils. If TFA intake is increased in blood then it also elevate the level of low-density lipoprotein and greater the risk of coronary diseases and also decreases high density lipoprotein.

Arthrosclerosis is a provocative process that happens due to the gathering of lipid within the arterial wall. It rises when plasma cholesterol level in blood is elevated which changes arterial endothelial cells permeability that store cholesterol containing low density lipoproteins (LDL) bind to the extracellular proteoglycan rich matrix accumulate into the arterial wall. Monocytes circulating also attach to endothelial cells that provide adhesion molecules, like vascular adhesion which provide movements to monocytes via diapedesis between endothelial junctions and reside in sub endothelial space, monocytes converted into macrophages and foamy macrophages [4]. The activated endothelium which have adhesion molecules that is early phase in artheosclerosis, allow lymphocytes such as monocytes and $\mathrm{T}$ cells to attach wall of blood vessel and penerate into inner dendritic cell, B cells,neutrophils and mast cells also locate in lesions. The abundant immune competent cells are present largely in artheosclerotic lesion producing cytokines. When plaque occur in blood vessel it lower blood flow and induce CVD, In artherothrombosis when thrombus were damaged, through the impact of chemokines and proinflammatory protein cytokines on the fibrous cap. CVD induced and prothrombotic material is attached to coagulation that inhibits the flow of blood. With the modification of CVD it also lower the risk of its related dieases like hypertension; and diabetes [5].

Consumption of dietary polyunsaturated fatty acid (PUFA) and monounsaturated fatty acid (MUFA) play an important role in production and oxidation of SFA and also decrease fatty content in hypercholesterolemia. They also have inflammatory properties and beneficial in managing of autoimmune diseases. In insulin resistance, Omega 6 fatty acid is important in controlling and also play important role in preventing, coronary heart diseases, depression, aging and cancer [6]. The objective of study involves evaluation of commonly used fats and oil to investigate its effect on lipid profile through in vivo studies. So, this research provide useful information regarding the choices of fats and oils for healthy weight gain and disease prevention specifically for hypercholesterolaemic patients in future.

\section{Material and methods}

The research work was carried out in the National Institute of Food Science and Technology, University of Agriculture, Faisalabad. For that purpose, Margarine, Butter, Hydrogenated ghee and Olive oil were procured from local market.

\subsection{Experimental animal model}

In this research role of commonly used fats and oil were assessed on hypercholesterolaemia by planning experimental model. For this purpose, 30 rats were attained and housed in animal room of National Institute of Food Science and Technology, University of Agriculture, Faisalabad. Rats were adapted for a period of 1 week by the providing regular diet and water ad libitum.

\subsection{Composition of experimental diet and induction of hypercholesterolaemia}

Induction of hypercholesterolaemia was done by feeding high cholesterol diet ( $2 \%$ cholesterol) with some modification according to procedure reported by [7]. Normal diet was containing $75 \%$ carbohydrates, $15 \%$ protein, $10 \%$ fat; high cholesterol diet was containing $75 \%$ carbohydrates, $15 \%$ protein, $8 \%$ fat and $2 \%$ cholesterol of total Kcal of the diet.

\subsection{Experimental protocol}

There were six groups $G_{0}, G_{1}, G_{2}, G_{3}, G_{4}$, $G_{5}$ in which animal Sprague dawly rat were distributed and each of them consisting of five rats. In experimental study, rats were attained for 1 week, 2 weeks for induction of 
hypercholesterolemia and 6 weeks for study period. Normal diet alone was given to $\mathrm{G}_{0}$ categorized as normal group. For the purpose of inducing hypercholesterolaemia, high cholesterol was given to rest of the groups and after 2 weeks baseline measurements were recorded to observe the level of hypercholesterolaemic induction. High cholesterol diet alone provided to $\mathrm{G}_{1}$. Along with high cholesterol diet, margarine, butter, vanaspati ghee, olive oil were given to $\mathrm{G}_{2}$, $\mathrm{G}_{3}$, $\mathrm{G}_{4}, \mathrm{G}_{5}$ to evaluate the effect of commonly used fats and oil on hypercholesterolaemic rats.

Table 1 Treatment Plan

\begin{tabular}{lll}
\hline Groups & Description & Treatments \\
\hline G0 & Normal & Normal Diet \\
G1 & Control & High cholesterol Diet \\
G2 & Margarine & High cholesterol Diet + Margarine \\
G3 & Butter & High cholesterol Diet + Butter \\
G4 & Vanaspati ghee & High cholesterol Diet + Vanaspati ghee \\
G5 & Olive oil & High cholesterol Diet + Olive oil \\
\hline
\end{tabular}

\subsection{Biological assessment}

Diet consumption was evaluated on weekly basis. Body weight gain was measured after 15 days interval. Blood samples were assembled and resultant parameters were considered to evaluate serum lipid profile of hypercholesterolaemic rats [8].

\subsection{Serum Lipid Profile}

Serum lipid profile of rats include low density lipoprotein (LDL), High density lipoprotein (HDL), cholesterol and triglycerides measured by following methods and their detail is given below:

\subsubsection{Cholesterol}

Serum cholesterol of rats was measured by using the following protocoal of [9].

\subsubsection{High and low density lipoprotein}

High density lipoprotein (HDL) and low density lipoproteins (LDL) in serum samples were deliberate by following the Dos Santos [10].

\subsubsection{Triglycerides}

Triglycerides in serum sample were calculated by Dixon and O'brien [11].

\subsection{Hematological analysis}

Red blood cells (RBC), white blood cell (WBC) and platelets count estimation was carried out following the method of Kamatani [12].

\subsection{Statistical analysis}

To assess the level of significance all factors of study was measured accurately and statistically via one-way factorial by Tukey test. Set standard for statistical significance in all cases was considered as $p<0.05$. Statistical analysis was done via analytical software Statistix 8.1 Al-Okbi [13].

\subsection{Statistical analysis}

To assess the level of significance all factors of study was measured accurately and statistically via one-way factorial by Tukey test. Set standard for statistical significance in all cases was considered as $p<0.05$. Statistical analysis was done via analytical software Statistix $8.1 \mathrm{Al}-\mathrm{Okbi}$ [13]. 


\section{Results}

Table 1 compares the serum lipid profiles of normal rats (rats fed with normal diet) with rats fed with High cholesterol diet and rats fed with (fats and oil besides HCD) along with different fats and oil. When rats were fed with high-cholesterol diet, there was a significant increase in the serum total cholesterol level and LDL cholesterol levels (both) in all groups. LDL level increased in fats treated group (margarine, butter, vanaspati ghee) and cholesterol fed rats. Further, triglyceride level showed a significant rise in the cholesterol fed rat group and also in (rats fed with normal chow diet versus rats fed with normal chow diet + different oils). Whereas a significant change was observed in margarine, vanaspati ghee, and butter fed group. HDL level decreased in all fats treated group.

Table 2 compares the feed intake body weight gain and hematological parameters of control rats (rats fed with normal diet) with rats fed with HCD and rats fed with high cholesterol diet (HCD) and different fats and oil group.Feed intake in all groups is significant and body weight gain is also significant in all groups elevated weight gain is noticed in vanaspati ghee fed group and lower weight gain is noticed in olive oil feed group. RBC level decreased significantly in all fats and oil treated groups and in high cholesterol diet group. WBC and platelets significantly decrease in all fats and oil treated groups also in high cholesterol group.

Table 2 Lipid profile parameters in control and different treated groups

\begin{tabular}{lllll}
\hline Groups (n= 5) & $\begin{array}{l}\text { TAG } \\
\text { (mg/dL) }\end{array}$ & $\begin{array}{l}\text { Total } \\
\text { Cholesterol } \\
\text { (mg/dl) }\end{array}$ & $\begin{array}{l}\text { HDL } \\
\text { Cholesterol } \\
\text { (mg/dL) }\end{array}$ & $\begin{array}{l}\text { LDL Cholesterol } \\
\text { (mg/dL) }\end{array}$ \\
\hline Group 0 & $79.6 \pm 2.71$ & $109.67 \pm 3.9$ & $23.5 \pm 0.74$ & $29.42 \pm 2.81$ \\
Group 1 & $150.5 \pm 4.4$ & $173.2 \pm 3.88$ & $23.4 \pm 0.75$ & $105.4 \pm 5.3$ \\
Group 2 & $126.5 \pm 0.84$ & $177.6 \pm 4.25$ & $23.7 \pm 0.7$ & $96.6 \pm 18.14$ \\
Group 3 & $130.2 \pm 1.24$ & $179.5 \pm 5.9$ & $23.6 \pm 0.65$ & $95.4 \pm 6.12$ \\
Group 4 & $134.86 \pm 18.9$ & $180.3 \pm 6.92$ & $23.5 \pm 0.93$ & $100.5 \pm 4.7$ \\
Group 5 & $95.6 \pm 4.38$ & $143.44 \pm 7.45$ & $23.5 \pm 0.78$ & $75.66 \pm 5.31$ \\
\hline \multicolumn{5}{l}{}
\end{tabular}

Table 3 Feed intake, body weight gain and hematological parameters in control and different treated groups

\begin{tabular}{llllll}
\hline $\begin{array}{l}\text { Groups } \\
\mathbf{n = 5}\end{array}$ & $\begin{array}{l}\text { Feed intake } \\
\mathbf{2 0} \text { g/per } \\
\text { body weight }\end{array}$ & $\begin{array}{l}\text { Body weight } \\
\text { gain } \\
\text { g/month }\end{array}$ & $\begin{array}{l}\mathbf{R B C} \\
\mathbf{\times 1 0 ^ { 6 } / \boldsymbol { \mu L }}\end{array}$ & $\begin{array}{l}\mathbf{W B C} \\
\times \mathbf{1 0} / \mathbf{L}\end{array}$ & $\begin{array}{l}\text { Platelets } \\
\times \mathbf{1 0} / \mathbf{L}\end{array}$ \\
\hline Group 0 & $20.4 \pm 3.68$ & $97.6 \pm 9.73$ & $7.08 \pm 0.015$ & $10220 \pm 153.18$ & $150850 \pm 134.16$ \\
Group 1 & $22.4 \pm 3.68$ & $150.5 \pm 3.47$ & $6.48 \pm 0.23$ & $43790 \pm 101.55$ & $4222960 \pm 1137.2$ \\
Group 2 & $18.4 \pm 3.08$ & $122.8 \pm 2.52$ & $6.56 \pm 0.015$ & $10010 \pm 77.18$ & $382790 \pm 375101.98$ \\
Group 3 & $21.9 \pm 3.86$ & $142 \pm 1.18$ & $6.17 \pm 0.225$ & $34180 \pm 527.3$ & $471580 \pm 475.34$ \\
Group 4 & $21.7 \pm 4.12$ & $142.8 \pm 3.69$ & $6.55 \pm 0.225$ & $10280 \pm 62.705$ & $422190 \pm 403.6$ \\
Group 5 & $14.9 \pm 1.73$ & $129.2 \pm 2.75$ & $6.56 \pm 0.015$ & $10310 \pm 69.2$ & $470690 \pm 2170.6$ \\
\hline
\end{tabular}

\section{Discussion}

Hypercholesterolemia is one of the predominant risk factor for development of atherogensis and coronary heart diseases. In development and progress of atherogensis reactive oxygen species (ROS) play a major role causing the conversion of LDL to oxidized LDL, which is consumed by macrophages. If macrophages increase the feed of oxidized LDL causes the formation of atherosclerotic plaques. Excessive uptake of modified macrophages causes the transformation of atherosclerotic plaques. Vulnerability of LDL to oxidation depends both on concentration of prooxidant stimuli. 
Administration of different fats and oil in rat daily for 2 months found a significant change in lipid profile as evident from the results of this study.

Lipid profile is main indicator of atherosclerosis. Our study is first to investigate the effects of commonly used fats and oil in sprague dawly rats.

Rats fed on high cholesterol diet and vegetable oils show a significant increase in lipid profile compared to the control. There is a significant increase in serum total cholesterol, LDL and triglycerides in all groups. Butter increases the LDL cholesterol significantly more than other group treated. A previous study evaluated different oils such as butter, olive oil and coconut oil. Results from review study indicate that butter consumption was significantly increased LDL-C concentration as compared with olive oil and coconut oil [14].

Margarine and vanaspati ghee also increased LDL significantly; but olive oil did not change its level. Furthermore, total cholesterol level and LDL cholesterol found to be increased in all fats and oil treated groups. The increase in TAG found in the olive oil group. Addition of commonly used fats and oil fed with high cholesterol diets has a positive influence on lipid metabolism. In the present study, effect of commonly used fats and oil on lipid profile was observed. Increased level of lipid profile was found in hypercholesterolaemic rats. Simultaneous administration of commonly used fats and oil with high cholesterol diet caused a significant increase in serum total cholesterol, LDL and Triglycerides.

In butter saturated fatty acid (SFA) and Trans fatty acids (TFA) are atherothrombic and associated with myocardial infarction MI and CHD more than other CVD outcomes [15], so butter increases the triglyceride (TC), LDL cholesterol and triglyceride treated group.

Margarine have high level of trans fatty acid so trans fatty acids and saturated fat produce similarly high levels of LDL cholesterol in plasma, but trans fatty acids also lower HDL cholesterol. Another study inspected to determine oxidation of vanaspati ghee while heating free radicals formed and examine its effect on lipid profile in vitro studies. It was indicated that vanaspati ghee have significant effect on lipid profile and peroxidation effect on tissue level [16].

Vegetable oils, such as olive and soya oils, are recommended for consumption due to their high content of monounsaturated and polyunsaturated fatty acids (MUFA) and (PUFA) [17]. A study comparing the impact of sunflower, fish, and virgin olive oils on the progression of experimental atherosclerosis in rabbits found that extra virgin olive oil; and to a lesser extent, fish oil, stops its progression. In another study, the aorta and coronary arteries of albino rats administered olive oil showed less atheromatous lesions compared with animals fed on corn oil or peanut oil.

At the end of study haematological analysis was done in which RBC, WBC and Platelets were determined by Kamatani [12]. The external structure of cholesterol constitutes the non-polar, hydrophobic lipid of the the enveloping layer of RBC membrane. When cholesterol level increases in blood it affects the fluidity of the membrane decreases and its outer lipid shell stiffens. Previous studies have shown that if blood cholesterol increases in blood that reduced the 02 transport: in addition the haemoglobin curve also shifted to the left. Early studies demonstrated that high blood cholesterol concentrations were associated with reduced blood 02 transport; in essence, the hemoglobin dissociation curve was shifted to the left. Present studies have shown that the cholesterol also affects hematological membrane barrier to 02 diffusion delayed 02 entry into the RBC during saturation and delayed 02 release from the RBC during desaturation. Results indicated that consumption of fats significantly increase the RBC, WBC and Platelets [18]. Enrichment of food with complementary antioxidants would be a best option to provide additional protective effects [19]. Olive oil scavenges free radicals and has enough for inflammation and auto immune diseases prevention [20].

\section{Conclusion}

Rat's food intake and growth with both levels of added oil was satisfactory. Overall it is summarized that ingestion of commonly used fats and oil elevated cholesterol level and it increased the risk of atherosclerosis. Butter increases the lipid profile of hypercholesterolaemic rats than any other group. Vanaspati ghee also increased total cholesterol (TC), triglyceride and little impact on HDL. Olive oil that does not increased cholesterol so its use is beneficial for health because it contain high content of MUFA and PUFA and also have antioxidant property to prevent atherosclerosis, but further research should be conducted in order to assess the effect of others fats and oil that used in everyday life. 


\section{Compliance with ethical standards}

\section{Acknowledgments}

All author would gratefully thank National Institute of Food Science and Technology, University of Agriculture, Faisalabad and Department of Zoology, University of the Punjab, Lahore for valuable support in carrying out this work.

\section{Disclosure of conflict of interest}

All authors declare that they have no conflict of interest.

\section{Statement of ethical approval}

This study was ethically approved by ORIC, University of Agriculture, Faisalabad, Pakistan.

\section{References}

[1] O'flaherty M, Buchan I and Capewell S. (2013). Contributions of treatment and lifestyle to declining CVD mortality: why have CVD mortality rates declined so much since the 1960s? Heart, 99, 159 -162.

[2] Oladapo 00, Ojora KA, Quadri OM and Ajani RS. (2017). Lipidemic effects of common edible oils and risk of atherosclerosis in diabetic Wistar rats. Journal of ARYA atherosclerosis, 13, 14-19.

[3] Nirmala KS, Bhat MS and Sahajananda H. (2016). Effect of two types of dietary ghee on serum lipid levels in rats. Journal of Evolution of Medical and Dental Sciences, 5, 3240-3244.

[4] Sakakura K, Nakano M, Otsuka F, Ladich E, Kolodgie FD and Virmani R. (2013). Pathophysiology of atherosclerosis plaque progression. Heart, Lung and Circulation, 22, 399-411.

[5] Frostegård J. (2013). Immunity, atherosclerosis and cardiovascular disease. BMC Medicine, 11, 117-132.

[6] Kozłowska-Wojciechowska M, JastrzęBska M, Naruszewicz M, and FoltyńSka. (2003). Impact of margarine enriched with plant sterols on blood lipids, platelet function. The Journal of Metabolism, 52, 1373-1378.

[7] Qian W, Hasegawa J, Tsuno S, Endo Y, Matsuda A and Miura N. (2014). Effects of kampo formulas on the progression of hypercholesterolemia and Fatty liver induced by high-cholesterol diet in rats. Yonago Acta Medica, 57, 147-157.

[8] Manjeshwar PR, D'souza AE, Bhat S, Bhat KMR and Arunkumar N. (2017). Effect of various vegetable oils on the lipid profile and antioxidant status in hypercholesterolaemic wistar rats-a comparative study. Journal of Evidence Based Medicine and Healthcare, 4, 778-782.

[9] Durrington PN. (2002). Can measurement of apolipoprotein B replace the lipid profile in the follow-up of patients with lipoprotein disorders? Journal of Clinical Chemistry, 48, 401-402.

[10] Dos Santos RE, Aldrighi JM, Lanz JR, Ferezin, PC and Marone MM. (2005). Relationship of body fat distribution by waist circumference, dual-energy X-ray absorptiometry and ultrasonography to insulin resistance by homeostasis model assessment and lipid profile in obese and non-obese postmenopausal women. Journal of Gynecological endocrinology, 21, 295-301.

[11] Dixon JB and O'brien PE. (2002). Lipid profile in the severely obese: changes with weight loss after lap-band surgery.the Journal of Obesity research, 10, 903-910.

[12] Kamatani Y, Matsuda K, Okada Y, Kubo M, Hosono N, Daigo Y, Nakamura and Kamatani N. (2010). Genomewide association study of hematological and biochemical traits in a Japanese population. Journal of Nature genetics, 42, 210-215.

[13] Al-Okbi SY, Mohamed DA, Hmed TE and Edris AE. (2013). Potential protective effect of Nigella sativa crude oils toward fatty liver in rats European. European Journal of Lipid Science and Technology, 115, 774-782.

[14] Khaw KT, Sharp SJ, Finikarides L, Afzal I, Lentjes M, Luben R. and Forouhi NG. (2018). Randomised trial of coconut oil, olive oil or butter on blood lipids and other cardiovascular risk factors in healthy men and women. BMJ open, 8, 20-167. 
[15] Hur SJ, Nam M, Du Km, Williamsonand M and Ahn DU. (2005). Effect ofdietary fats on blood cholesterol and lipid and the development of atherosclerosis in rabbits. Journal of Nutrition Research, 25, 925-935.

[16] Zeb A and Uddin I. (2017). The Coadministration of Unoxidized and Oxidized Desi Ghee Ameliorates the Toxic Effects of Thermally Oxidized Ghee in Rabbits. Journal of nutrition and metabolism, 2017, 1-7.

[17] Shahid SU, Cooper JA, Beaney KE, Rehman KLi A and Humphries SE. (2017). Genetic risk analysis of coronary artery disease in Pakistani subjects using a genetic risk score of 21 variants. Journal of Atherosclerosis, 258, 17.

[18] Kellogg TA, Bantle JP, Leslie DB, Redmond JB, Slusarek B, Swan T, Buchwald H and Ikramuddin S. (2008). Postgastric bypass hyperinsulinemic hypoglycemia syndrome: characterization and response to a modified diet. Journal of Surgery for Obesity and Related Diseases, 4, 492-499.

[19] Visioli F, Galli C, Galli G and Caruso D. (2002). Biological activities and metabolic fate of olive oil phenols. European Journal of Lipid Science and Technology, 104, 677-684.

[20] Nirmala KS, Bhat MS and Sahajananda H. (2016). Effect of two types of dietary ghee on serum lipid levels in rats. Journal of evolution of Medical and Dental Sciences, 5, 3240-3244.

\section{How to cite this article}

Javaid F, Zahoor T, Nazeer S, Ramzan U and Matloob A. (2019). Biological study on the impact of commonly used commercial fats and oil and threats of atherosclerosis GSC Biological and Pharmaceutical Sciences, 8(1), 98-104. 